\title{
FUNDAMENTAL SOLUTIONS FOR IDEAL FLUIDS IN UNIFORM MOTION*
}

\author{
BY \\ LAZÅR DRAGOS \\ University of Bucharest
}

\begin{abstract}
In this paper the perturbations produced by mass, momentum and energy sources in the uniform flow of an ideal fluid are determined. The case of instantaneous sources is considered. The matrix solutions thus determined are by definition the fundamental matrices of the systems of equations of fluid mechanics. As an application, the perturbations caused by sources acting permanently for $t>0$ as well as the perturbations produced by sources moving along the direction of the free flow or perpendicular to this direction are determined.
\end{abstract}

1. In [1] we have determined the perturbations produced by mass, momentum and energy sources in an ideal (or viscous) fluid at rest. In [2] we have constructed the fundamental matrix for this fluid.

Extending the methods of [1] and [2], in this paper we shall determine the perturbations induced by mass, momentum and energy sources in an ideal fluid in uniform motion. We shall build up the fundamental matrix in this case and we shall apply the results to problems of great practical interest. The case of dissipative fluids will be analysed in [3].

We assume that the basic state of the fluid is characterized by the constant parameters $\rho_{0}, \mathbf{V}_{0}, p_{0}$ and $T_{0}\left(p_{0}=\rho_{0} R T_{0}\right)$ and we shall choose the reference system such that the $O x_{1}$ axis has the direction of the vector $\mathbf{V}_{0}$. At an arbitrary time $t>0$, the fluid state will be characterized by the variables

$$
\rho=\rho_{0}\left(1+\rho^{*}\right), \quad \mathbf{V}=V_{0}\left(\mathrm{i}_{1}+\mathbf{v}\right), \quad p=p_{0}+\rho_{0} V_{0}{ }^{2} p^{*}
$$

and $T=T_{0}\left(1+T^{*}\right)$, the quantities $\rho_{0} \rho^{*}, V_{0} \mathbf{v}, \rho_{0} V_{0}^{2} p^{*}$ and $T_{0} T^{*}$ representing the perturbations induced by the sources. The functions $\rho, \mathbf{V}, p$ and $T$ must satisfy Eqs. (1)-(4) of [1] in which the dissipative terms are neglected. In order that the equations be treated in a unitary way, we shall assume that the sources are acting in the origin of the system of coordinates. The problem concerning the sources acting in different points does not present additional difficulties [1]. Also, for the sake of uniformity, we shall introduce the dimensionless variables $x_{i}{ }^{*}$ and $t^{*}$ defined by the relations $x_{i}=L_{0} x_{i}{ }^{*}, V_{0} t=L_{0} t^{*}$, where $L_{0}$ in an arbitrary characteristic length, and we shall drop the sign *. Linearizing the motion equations in order to determine the perturbation, we obtain the following system:

* Received January 30, 1978; revised version received May 4, 1978. 


$$
\begin{gathered}
\partial_{t} \rho+\partial_{1} \rho+\operatorname{div} \mathbf{v}=f_{0} \delta(t, \mathbf{x}), \delta(t, \mathbf{x})=\delta(t) \delta(\mathbf{x}), \\
\partial_{t} v_{i}+\partial_{1} v_{i}+\partial_{i} p=f_{i} \delta(t, \mathbf{x})(i=1,2,3), \\
\partial_{t} T+\partial_{1} T+(\gamma-1) \operatorname{div} \mathbf{v}=\left(\gamma f_{4}-f_{0}\right) \delta(t, \mathbf{x}), \\
\gamma M^{2} p=\rho+T,
\end{gathered}
$$

where $f_{j}(j=0,1,2,3,4)$ are expressed with the aid of the intensities $m_{0}, f^{0}$ and $Q_{0}$ of the mass, momentum and energy sources using the following expressions:

$$
\begin{gathered}
\rho_{0} L_{0}{ }^{3} f_{0}=m_{0}, \quad \rho_{0} L_{0}{ }^{3} V_{0} f_{i}=f_{i}{ }^{0}-\delta_{i 1} m_{0} V_{0}, \\
c_{v} \rho_{0} L_{0}{ }^{3} T_{0} \gamma f_{4}=Q_{0}+\frac{1}{2} m_{0} V_{0}{ }^{2}-f_{1}{ }^{0} V_{0},
\end{gathered}
$$

where $\delta_{i j}$ is the Kronecker delta. As usual, we have used the notation $\gamma c_{v}=c_{p}, a_{0} M=V_{0}$, $a_{0}{ }^{2}=\gamma R T_{0}$, where $c_{v}$ and $c_{p}$ are the specific heats of the fluid, while $R$ is the universal constant of gases. Adding $(2)_{1}$ to $(2)_{3}$ and taking into account $(2)_{4}$, we get

$$
M^{2}\left(\partial_{t}+\partial_{1}\right) p+\operatorname{div} \mathbf{v}=f_{4} \delta(t, \mathbf{x})
$$

Therefore we shall consider Eqs. $(2)_{1},(2)_{2}$ and (3) with the unknowns $\rho, v_{i}$ and $p$. These equations will have to be integrated all over the space in which the space conditions are

$$
\lim _{\mathbf{x}: \rightarrow \infty}\left(\rho, v_{i}, p\right)=0
$$

and the temporal conditions

$$
\rho=0, \quad v_{i}=0, \quad p=0, \quad t \leq 0 .
$$

Eq. (4) expresses the damping condition of the perturbation at infinity and (5) the condition of nonexistence of the perturbation before the source begins to act.

Hereafter we shall use the matrix method in order to determine the solution of the system (2), (3); in this way we have the possibility of putting into evidence the basic structure of the solution and at the same time of writing the general solution of the system (2), (3) in the case in which in the right-hand side of the equalities the arbitrary functions $f(t, \mathbf{x})$ would appear. Introducing the matrices

$$
\begin{aligned}
& A_{1}=\left(\begin{array}{lllll}
1 & 1 & 0 & 0 & 0 \\
0 & 1 & 0 & 0 & M^{-2} \\
0 & 0 & 1 & 0 & 0 \\
0 & 0 & 0 & 1 & 0 \\
0 & 1 & 0 & 0 & 1
\end{array}\right), A_{2}=\left(\begin{array}{lllll}
0 & 0 & 1 & 0 & 0 \\
0 & 0 & 0 & 0 & 0 \\
0 & 0 & 0 & 0 & M^{-2} \\
0 & 0 & 0 & 0 & 0 \\
0 & 0 & 1 & 0 & 0
\end{array}\right), \\
& A_{3}=\left(\begin{array}{lllll}
0 & 0 & 0 & 1 & 0 \\
0 & 0 & 0 & 0 & 0 \\
0 & 0 & 0 & 0 & 0 \\
0 & 0 & 0 & 0 & M^{-2} \\
0 & 0 & 0 & 1 & 0
\end{array}\right), \quad V=\left(\begin{array}{l}
\rho \\
v_{1} \\
v_{2} \\
v_{3} \\
M^{2} p
\end{array}\right), \quad f=\left(\begin{array}{l}
f_{0} \\
f_{1} \\
f_{2} \\
f_{3} \\
f_{4}
\end{array}\right),
\end{aligned}
$$

we can write the system consisting of Eqs. $(2)_{1},(2)_{2}$ and (3) as follows:

$$
\partial_{t} V+A_{k} \partial_{k} V=f \delta(t, \mathbf{x})
$$

The fundamental matrix $K(t, \mathbf{x})$ introduced by the formula $V=K f$ will be determined by equation

$$
\partial_{t} K+A_{k} \partial_{k} K=E \delta(t, \mathbf{x})
$$


where $E$ is the unit matrix with $5 \times 5$ elements, and by the conditions

$$
K(t \leq 0, \mathbf{x})=0, K(t, \infty)=0
$$

with the usual notations.

2. As is already known (Duhamel's principle, see also [4]), the solution of the problem (7), (8) is of the form $K=H(t) K^{*}(t, \mathbf{x})$ where $H(t)$ is the Heaviside function and $K^{*}$ the matrix defined by equation

$$
\partial_{t} K^{*}+A_{k} \partial_{k} K^{*}=0
$$

and by the conditions

$$
K^{*}(0, \mathbf{x})=E \delta(\mathbf{x}), \quad K^{*}(t, \infty)=0 .
$$

For

$$
\bar{K}=\mathbf{f}\left(K^{*}\right)=\iiint_{-\infty}^{+\infty} K \exp i\left(\alpha_{1} x_{1}+\alpha_{2} x_{2}+\alpha_{3} x_{3}\right) d x_{1} d x_{2} d x_{3}
$$

we obtain

$$
\dot{\bar{K}}=\bar{Q} \bar{K}, \quad \bar{K}(0, \boldsymbol{\alpha})=E
$$

where

$$
\overline{\mathrm{Q}}=i \alpha_{k} A_{k}=i\left(\begin{array}{ccccc}
\alpha_{1} & \alpha_{1} & \alpha_{2} & \alpha_{3} & 0 \\
0 & \alpha_{1} & 0 & 0 & \alpha_{1} M^{-2} \\
0 & 0 & \alpha_{1} & 0 & \alpha_{2} M^{-2} \\
0 & 0 & 0 & \alpha_{1} & \alpha_{3} M^{-2} \\
0 & \alpha_{1} & \alpha_{2} & \alpha_{3} & \alpha_{1}
\end{array}\right)
$$

The solution of Eq. (12) is

$$
\bar{K}=E \exp (\overline{\mathbb{Q}} t) .
$$

In order to determine the function $\exp (\bar{Q} t)$ we use the method of the minimal polynomial. The roots of the characteristic polynomial of the matrix $Q$ are

$$
\lambda_{1}=i \alpha_{1}, \lambda_{2}=i\left(\alpha_{1}+|\boldsymbol{\alpha}| M^{-1}\right), \lambda_{3}=i\left(\alpha_{1}-|\alpha| M^{-1}\right)
$$

where multiplicity of the first root is equal to three. Since the minimal polynomial $m(\lambda)$ has the expression

$$
\begin{gathered}
m(\lambda)=\left(\lambda-\lambda_{1}\right)\left(\lambda-\lambda_{2}\right)\left(\lambda-\lambda_{3}\right)=\lambda^{3}-a_{2} \lambda^{2}-a_{1} \lambda-a_{0}, \\
a_{2}=3 i \alpha_{1}, a_{1}=3 \alpha_{1}^{2}-|\boldsymbol{\alpha}|^{2} M^{-2}, a_{0}=i \alpha_{1}\left(|\boldsymbol{\alpha}|^{-2} M^{-2}-\alpha_{1}^{2}\right),
\end{gathered}
$$

it follows that the solution of system (12) is

$$
\bar{K}=E g_{0}(t)+\bar{a} g_{1}(t)+\bar{Q}^{2} g_{2}(t)
$$

where $g_{2}, g_{0}$ and $g_{1}$ are determined by the equations

$$
\begin{gathered}
g_{2}^{\prime \prime \prime}-a_{2} g_{2}^{\prime \prime}-a_{1} g_{2}^{\prime}-a_{0} g_{2}=0, \quad g_{2}(0)=g_{2}^{\prime}(0)=0, \quad g_{2}^{\prime \prime}(0)=1, \\
g_{0}^{\prime}=a_{0} g_{2}, \quad g_{0}(0)=1 ; \quad g_{1}^{\prime}=a_{1} g_{2}+g_{0}, g_{1}(0)=0,
\end{gathered}
$$


We obtain

$$
\begin{aligned}
& g_{0}=\left[1+\frac{\alpha_{1}^{2} M^{2}}{|\boldsymbol{\alpha}|^{2}}\left(\cos \frac{|\boldsymbol{\alpha}|}{M} t-1\right)-\frac{i \alpha_{1} M}{|\boldsymbol{\alpha}|} \sin \frac{|\boldsymbol{\alpha}|}{M} t\right] \exp \left(i \alpha_{1} t\right), \\
& g_{1}=\left[\frac{M}{|\alpha|} \sin \frac{|\alpha|}{M} t+\frac{2 i \alpha_{1} M^{2}}{|\boldsymbol{\alpha}|^{2}}\left(\cos \frac{|\boldsymbol{\alpha}|}{M} t-1\right)\right] \exp \left(i \alpha_{1} t\right), \\
& g_{2}=\frac{M^{2}}{|\boldsymbol{\alpha}|^{2}}\left(1-\cos \frac{|\boldsymbol{\alpha}|}{M} t\right) \exp \left(i \alpha_{1} t\right) .
\end{aligned}
$$

Taking into account formulae (A.1)-(A.3) in the Appendix, we obtain the following fundamental matrix:

$$
\begin{aligned}
K^{*} & =E \delta\left(t-x_{1}\right) \delta\left(x_{2}\right) \delta\left(x_{3}\right)+\frac{M^{2}}{4 \pi t}\left(E \partial_{1}+Q\right) \delta\left\{M^{-1} t-\left[\left(x_{1}-t\right)^{2}+r_{0}^{2}\right]^{1 / 2}\right\} \\
& +\frac{M^{2}}{4 \pi}\left(E \partial_{11}^{2}+2 Q \partial_{1}+Q^{2}\right) \frac{H\left\{M^{-1} t-\left[\left(x_{1}-t\right)^{2}+r_{0}^{2}\right]^{1 / 2}\right\}}{\left[\left(x_{1}-t\right)^{2}+r_{0}^{2}\right]^{1 / 2}},
\end{aligned}
$$

where the matrix-operator $Q$ is obtained from $\bar{Q}$ by substituting $-\partial_{j} \leftarrow i \alpha_{j}$. We have, for instance,

$$
M^{2} Q^{2}=\left(\begin{array}{lllll}
M^{2} \partial_{11}^{2} & 2 M^{2} \partial_{11}^{2} & 2 M^{2} \partial_{12}^{2} & 2 M^{2} \partial_{13}^{2} & \partial_{i i}^{2} \\
0 & \left(1+M^{2}\right) \partial_{11}^{2} & \partial_{12}^{2} & \partial_{13}^{2} & 2 \partial_{11}^{2} \\
0 & \partial_{12}^{2} & M^{2} \partial_{11}^{2}+\partial_{22}^{2} & \partial_{23}^{2} & 2 \partial_{12}^{2} \\
0 & \partial_{13}^{2} & \partial_{23}^{2} & M^{2} \partial_{11}^{2}+\partial_{33}^{2} & 2 \partial_{13}^{2} \\
0 & 2 M^{2} \partial_{11}^{2} & 2 M^{2} \partial_{12}^{2} & 2 M^{2} \partial_{13}^{2} & M^{2} \partial_{11}^{2}+\partial_{i i}^{2}
\end{array}\right),
$$

$\partial_{i i}^{2} \equiv \partial_{11}^{2}+\partial_{22}^{2}+\partial_{33}^{2}$.

3. As can be proved [2], the general solution of the equation

$$
\partial_{t} V+A_{k} \partial_{k} V=f(t, \mathbf{x})
$$

with the conditions

$$
V(0, \mathbf{x})=0, V(t, \infty)=0,
$$

is

$$
V(t, \mathbf{x})=\int_{0}^{t} K^{* *} \int d \tau
$$

where

$$
K^{* *} f=\iiint_{-\infty}^{+\infty} K^{*}(t-\tau, \mathbf{x}-\xi) f(\tau, \xi) d \xi, \quad d \xi \equiv d \xi_{1} d \xi_{2} d \xi_{3} .
$$

Taking into account the expression of the fundamental matrix (16), it follows that the general solution of Eq. (17) is 


$$
\begin{aligned}
& V(t, \mathbf{x})=\int_{0}^{t} f\left(t-\tau, x_{1}-\tau, x_{2}, x_{3}\right) d \tau \\
& +\frac{M^{2}}{4 \pi}\left(E \partial_{1}+Q\right) \int_{0}^{t} \frac{d \tau}{\tau} \iiint_{-\infty}^{+\infty} f(t-\tau, \mathbf{x}-\xi) \delta\left\{M^{-1} \tau-\left[\left(\xi_{1}-\tau\right)^{2}+\xi_{0}^{2}\right]^{1 / 2}\right\} d \xi \\
& +\frac{M^{2}}{4 \pi}\left(E \partial_{11}^{2}+2 Q \partial_{1}+Q^{2}\right) \int_{0}^{t} d \tau \iiint_{-\infty}^{+\infty} f(t-\tau, \mathbf{x}-\xi) \frac{H\left\{M^{-1} \tau-\left[\left(\xi_{1}-\tau\right)^{2}+\xi_{0}^{2}\right]^{1 / 2}\right\}}{\left[\left(\xi_{1}-\tau\right)^{2}+\xi_{0}^{2}\right]^{1 / 2}} d \xi
\end{aligned}
$$

where $\xi_{0}^{2}=\xi_{2}^{2}+\xi_{3}^{2}$.

As a first application we consider the case in which the uniform motion of the fluid is perturbed by sources which in the origin act permanently for $t>0$, therefore the case in which

$$
f(t, \mathbf{x})=f^{0} H(t) \delta(\mathbf{x})
$$

where $H(t)$ is, as throughout this paper, Heaviside's function. We obtain

$$
\begin{aligned}
& V(t, \mathbf{x})=f^{0}\left[H\left(x_{1}\right)-H\left(x_{1}-t\right)\right] \delta\left(x_{2}\right) \delta\left(x_{3}\right)+\frac{M^{2}}{4 \pi}\left(E \partial_{1}+Q\right) f^{0} \int_{0}^{t} \frac{d \tau}{\tau} \delta\left(M^{-1}-R_{\tau}\right) \\
& +\frac{M^{2}}{4 \pi}\left(E \partial_{11}^{2}+2 Q \partial_{1}+Q^{2}\right) f^{0} \int_{0}^{t} \frac{H\left(M^{-1} \tau-R_{\tau}\right)}{R_{\tau}} d \tau, \quad R_{\tau}=\left[\left(x_{1}-\tau\right)^{2}+r_{0}^{2}\right]^{1 / 2} .
\end{aligned}
$$

For the calculation of the integrals occurring here the zeros of the function $h(\tau)=M^{-1}$ $\tau-R_{\tau}$ will have to be determined. We have $h(0)<0, \lim _{\infty} h(\tau)=\left(1-M^{2}\right) \infty$ and

$$
\beta^{2} \tau_{ \pm}=-M^{2} x_{1} \pm M R, R \equiv\left(r^{2}-M^{2} r_{0}^{2}\right)^{1 / 2}, \beta^{2} \equiv 1-M^{2} .
$$

For $M<1, h(\tau)$ has a single zero, $\tau_{+}$. For $M>1, h(\tau)$ has two zeros if $h_{0}=h\left(\tau_{0}\right)>0$, where $\tau_{0}$ is the root of the derivative $h^{\prime}(\tau)$, and no zero if $h_{0}<0$. We obtain $M h_{0}=x_{1}-$ $r_{0} \beta_{1}, \beta_{1} \equiv\left(M^{2}-1\right)^{1 / 2}, \tau_{+}<\tau_{-}$and $\tau_{ \pm} h^{\prime}\left(\tau_{ \pm}\right)= \pm R$. At points $\tau_{+}$the function $h$ is increasing $\left(h^{\prime}>0\right)$ and at points $\tau_{-}$it is decreasing $\left(h^{\prime}<0\right)$. Using the formula [5]

$$
\delta(h(\tau))=\sum_{i} \frac{\delta\left(\tau-\tau_{i}\right)}{\left|h^{\prime}\left(\tau_{i}\right)\right|}
$$

where $\tau_{i}$ are the zeros of the function $h$, we deduce

$$
\begin{array}{rlrl} 
& =R^{-1} H\left(t-\tau_{+}\right), & & \text {if } M<1 \\
\int_{0}^{t} \frac{d \tau}{\tau} \delta\left(M^{-1} \tau-R_{\tau}\right) & =R^{-1}\left[H\left(t-\tau_{+}\right)+H(t-\tau)\right], & \text { if } M>1 \text { and } h_{0}>0 \\
& = & 0 & \text { if } M>1 \text { and } h_{0}<0 .
\end{array}
$$

We also have

$$
\begin{aligned}
\int_{0}^{t} \frac{H\left(M^{-1} \tau-R_{\tau}\right)}{R_{\tau}} d \tau & =H\left(t-\tau_{+}\right) \ln \left|\frac{x_{1}+R}{\left(x_{1}-t+R_{t}\right)(M+1)}\right|, \text { for }(M<1) \cup \\
& =H\left(t-\tau_{-}\right) \ln \left|\frac{x_{1}+R}{x_{1}-R}\right|, \quad \text { for }\left(M>1, h_{0}>0, t<\tau_{-}\right) \\
& =0, \quad \text { for }\left(M>1, h_{0}<0\right) .
\end{aligned}
$$

In this way the solution (20) is completely determined. 
4. As a second application let us determine the perturbation induced by sources moving uniformly in the flow of the free fluid. Let us assume for the beginning that the sources are moving along the direction of the free flow. This means that in (18) we must write

$$
f(t, \mathbf{x})=f^{0} \delta\left(x_{1}-k_{0} t\right) \delta\left(x_{2}\right) \delta\left(x_{3}\right)
$$

where $k_{0}$ is the ratio between the velocity of the sources and the velocity of the free flow; $k_{0}$ is a positive number if both velocities are oriented in the same direction and a negative number if the velocities have opposite directions. From (18) we get

$$
\begin{gathered}
V(t, \mathbf{x})=f^{0} \delta\left(x_{2}\right) \delta\left(x_{3}\right) \int_{0}^{t} \delta\left(x_{1}-k_{0} t+k \tau\right) d \tau+\frac{M^{2}}{4 \pi}\left(E \partial_{1}+Q\right) f^{0} \int_{0}^{t} \frac{d \tau}{\tau} \delta\left(M^{-1} \tau-R_{\tau}\right) \\
+\frac{M^{2}}{4 \pi}\left(E \partial_{11}^{2}+2 Q \partial_{1}+Q^{2}\right) f^{0} \int_{0}^{t} \frac{H\left(M^{-1} \tau-R_{\tau}\right)}{R_{\tau}} d \tau
\end{gathered}
$$

where

$$
\begin{aligned}
\int_{0}^{t} \delta\left(x_{1}-k_{0} t+k \tau\right) d \tau & =\frac{1}{k} \int_{0}^{t} \delta\left(\tau+\frac{x_{1}-k_{0} t}{k}\right) d \tau=\frac{1}{K} H\left(\frac{x_{1}}{k}-\frac{t}{k}\right)-\left(k_{0} \frac{t}{k}-\frac{x_{1}}{k}\right), \\
R_{\tau} & =\left[\left(x_{1}-k_{0} t+k \tau\right)^{2}+r_{0}^{2}\right]^{1 / 2}, \quad k=k_{0}-1 .
\end{aligned}
$$

The study of the zeros of the function $h(\tau)=M^{-1} \tau-R_{\tau}$ is carried out as shown above. We have $h(0)<0, h(\infty)=\left(1-k^{2} M^{2}\right)^{\infty}$;

$$
\tau_{ \pm}=\frac{k^{2} M^{2}\left(x_{1}-k_{0} t\right) \pm M R}{1-M^{2} k^{2}}, R=\left[\left(x_{1}-k_{0} t\right)^{2}+\left(1-M^{2} k^{2}\right) r_{0}^{2}\right]^{1 / 2} .
$$

If $k^{2} M^{2}<1$ then there is a single positive root, namely $\tau_{+}$. If $1<k^{2} M^{2}$ then there are two roots $\tau_{+}$and $\tau_{-}\left(\tau_{+}<\tau_{-}\right)$if $h_{0}>0$ and no root at all if $h_{0}<0$, where

$$
M h_{0}=-\frac{r_{0}}{|k|}\left(M^{2} k^{2}-1\right)^{1 / 2}-\frac{x_{1}-k_{0} t}{k} .
$$

Since we also have the relation $\tau_{ \pm} h^{\prime}\left(\tau_{ \pm}\right)= \pm R$, we obtain

$$
\begin{aligned}
\int_{0}^{t} \frac{d \tau}{\tau} \delta\left(M^{-1} \tau-R_{\tau}\right) & =R^{-1} H\left(t-\tau_{+}\right), \quad \text { if } k^{2} M^{2}<1 \\
& =R^{-1}\left[H\left(t-\tau_{+}\right)+H\left(t-\tau_{-}\right)\right], \quad \text { if } \quad\left(1<k^{2} M^{2}, \quad h_{0}>0\right) \\
& =0, \text { if } \quad\left(1<k^{2} M^{2}, h_{0}<0\right) \\
\int_{0}^{t} \frac{H\left(M^{-1} \tau-R_{\tau}\right)}{R_{\tau}} d \tau & =\frac{H\left(t-\tau_{+}\right)}{k} \ln \left|\frac{(1-k M)\left\{x_{1}-t+\left[\left(x_{1}-t\right)^{2}+r_{0}^{2}\right]^{1 / 2}\right\}}{x_{1}-k_{0} t+R}\right| \\
& \text { for } \quad\left(k^{2} M^{2}<1\right) \cup\left(1<k^{2} M^{2}, h_{0}>0, t<\tau_{-}\right) \\
& =\frac{H\left(t-\tau_{-}\right)}{k} \ln \left|\frac{x_{1}-k_{0} t-R}{f_{1}-k_{0} t+R}\right|, \quad \text { for } \quad\left(1<k^{2} M^{2}, \quad h_{0}<0\right) . \\
& =0, \quad \text { for } \quad\left(1<k^{2} M^{2}, \quad h_{0}<0\right) .
\end{aligned}
$$


Let us now determine the solution in the case in which the sources are moving perpendicular to the direction of the free flow. This means that we write

$$
f(t, \mathbf{x})=f^{0} \delta\left(x_{1}\right) \delta\left(x_{2}-k_{0} t\right) \delta\left(x_{3}\right)
$$

in (18). We get

$$
\begin{aligned}
V(t, \mathbf{x}) & =f^{0} \delta\left(x_{3}\right) \int_{0}^{t} \delta\left(x_{1}-\tau\right) \delta\left(x_{2}-k_{0} t+k_{0} \tau\right) d \tau \\
& +\frac{M^{2}}{4 \pi}\left(E \partial_{1}+Q\right) f^{0} \int_{0}^{t} \frac{d \tau}{\tau} \delta\left(M^{-1} \tau-R_{\tau}\right) \\
& +\frac{M^{2}}{4 \pi}\left(E \partial_{11}^{2}+2 Q \partial_{1}+Q^{2}\right) f^{0} \int_{0}^{t} \frac{H\left(M^{-1} \tau-R_{\tau}\right)}{R_{\tau}} d \tau
\end{aligned}
$$

where, this time,

$$
\begin{gathered}
\int_{0}^{t} \delta\left(x_{1}-\tau\right) \delta\left(x_{2}-k_{0} t+k_{0} \tau\right) d \tau=\delta\left(k_{0} x_{1}+x_{2}-k_{0} t\right) \quad \text { if }\left(0<x_{1}<t, 0<t-k_{0}{ }^{-1} x_{2}<t\right) \\
=0 \text { otherwise } \\
R_{\tau}=\left[\left(x_{1}-\tau\right)^{2}+\left(x_{2}-k_{0} t+k_{0} \tau\right)^{2}+x_{3}^{2}\right]^{1 / 2} .
\end{gathered}
$$

In this case, too, the zeros of the function $h(\tau)=M^{-1} \tau-R_{\tau}$ are determined. We obtain

$$
\tau_{ \pm}=\frac{-M^{2}\left[x_{1}-k_{0}\left(x_{2}-k_{0} t\right)\right] \pm M R}{1-k^{2} M^{2}}
$$

where

$$
\begin{aligned}
& R^{2}=M^{2}\left[x_{1}-k_{0}\left(x_{2}-k_{0} t\right)\right]^{2}+\left(1-k^{2} M^{2}\right) R_{0}^{2}, \\
& R_{0}^{2}=x_{1}^{2}+\left(x_{2}-k_{0} t\right)^{2}+x_{3}^{2}, \quad k^{2}=1+k_{0}^{2} .
\end{aligned}
$$

For $R$ and $k$ the positive determination is taken into account. If $k^{2} M^{2}<1$ then the function $h$ has a single zero $\tau_{+}$. If $1<k^{2} M^{2}$ and $h_{0}>0$, where

$$
k^{2} M h_{0}=x_{1}-k_{0}\left(x_{2}-k_{0} t\right)-\left(k^{2} M^{2}-1\right)^{1 / 2}\left\{k^{2} R_{0}^{2}-\left[x_{1}-k_{0}\left(x_{2}-k_{0} t\right)\right]^{2}\right\}^{1 / 2},
$$

then the function $h(\tau)$ has two zeros, $\tau_{+}$and $\tau_{-}\left(\tau_{+}<\tau_{-}\right)$. Finally, if $1<k^{2} M^{2}$ and $h_{0}<0$, the function $h(\tau)$ has no zero at all. Taking into account $\tau_{ \pm} h^{\prime}\left(\tau_{ \pm}\right)= \pm R$, it follows that

$$
\begin{aligned}
\int_{0}^{t} \frac{d \tau}{\tau} \delta\left(M^{-1} \tau-R_{\tau}\right) & =R^{-1} H\left(t-\tau_{+}\right), \quad \text { if } \quad k^{2} M^{2}<1 \\
& =R^{-1}\left[H\left(t-\tau_{+}\right)+H\left(t-\tau_{-}\right)\right], \quad \text { if } \quad\left(1<k^{2} M^{2}, h_{0}>0\right) \\
& =0, \quad \text { if } \quad\left(1<k^{2} M^{2}, h_{0}<0\right)
\end{aligned}
$$




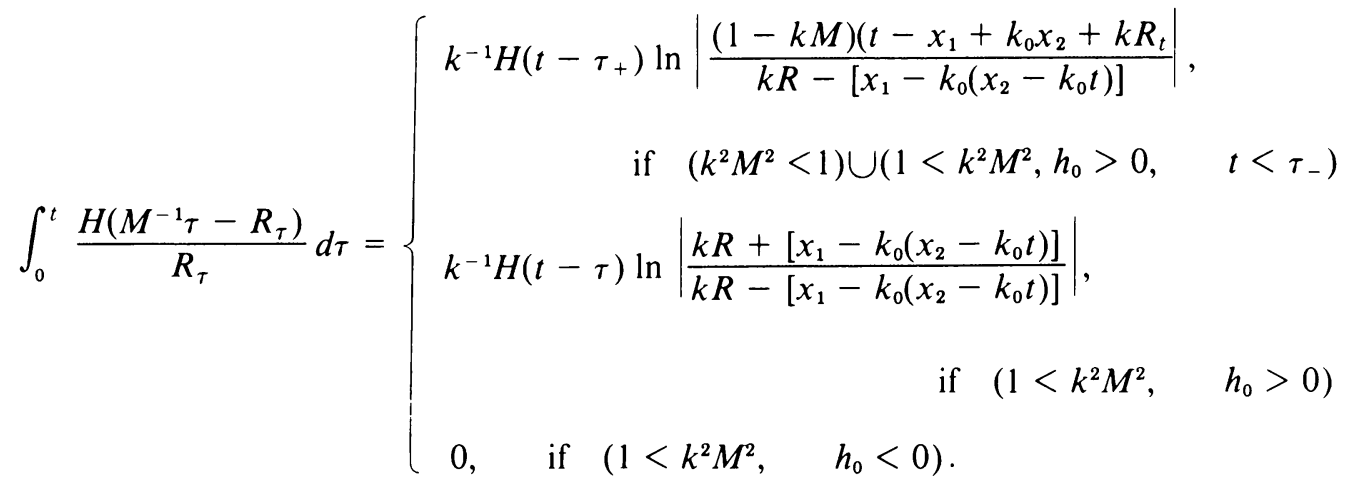

The solutions determined here have not only a theoretical importance but also a practical one. For example, the solution determined in Sec. 3 in the particular case $m_{0}=Q_{0}$ $=0$ gives the perturbation induced by a fixed body which can be assimilated to a point, in the presence of a uniform wind, and the solutions determined in Sec. 4 (for $m_{0}=Q_{0}=0$ ) give the perturbations produced at the leading edge of an aeroplane which moves in the direction of the wind (in the same direction or in the opposite direction to the wind) or perpendicular to the direction of the wind respectively.

Appendix. In Sec. 2 the following formulae are used:

$$
\begin{gathered}
f^{-1}\left(\exp \left(i \alpha_{1} t\right)\right)=\delta\left(t-x_{1}\right) \delta\left(x_{2}\right) \delta\left(x_{3}\right), \\
\tilde{f}-1\left(\frac{\exp \left(i \alpha_{1} t\right)}{|\boldsymbol{\alpha}|} \sin \frac{|\alpha|}{M} t\right)=\frac{M}{4 \pi t} \delta\left\{M^{-1} t-\left[\left(x_{1}-t\right)^{2}+r_{0}^{2}\right]^{1 / 2}\right\}, \\
\tilde{f^{-1}}\left(\frac{\exp \left(i \alpha_{1} t\right)}{|\boldsymbol{\alpha}|^{2}}\left(1-\cos \frac{|\alpha|}{M} t\right)\right)=\frac{H\left\{M^{-1} t-\left[\left(x_{1}-t\right)^{2}+r_{0}^{2}\right]^{1 / 2}\right\}}{4 \pi\left[\left(x_{1}-t\right)^{2}+r_{0}^{2}\right]^{1 / 2}} .
\end{gathered}
$$

Formula (A.1) is obvious. In order to deduce formula (A.2) we use cylindrical coordinates in the Fourier space: $\alpha_{1}=\alpha_{1}, \alpha_{2}=\rho \cos \theta, \alpha_{3}=\rho \sin \theta$ and cylindrical conrdinates in the physical space: $x_{1}=x_{1}, x_{2}=r_{0} \cos \phi, x_{3}=r_{0} \sin \phi$, as well as the formulae

$$
\begin{gathered}
\int_{0}^{\infty} \frac{J_{0}\left(\rho r_{0}\right) \rho \sin t M^{-1}\left(\rho^{2}+\alpha_{1}^{2}\right)^{1 / 2}}{\left(\rho^{2}+\alpha_{1}^{2}\right)^{1 / 2}} d \rho=M \frac{H\left(t-M r_{0}\right)}{\left(t^{2}-M^{2} r_{0}^{2}\right)^{1 / 2}} \cos \frac{\alpha_{1}}{M}\left(t^{2}-M^{2} r_{0}^{2}\right)^{1 / 2}, \\
\int_{-\infty}^{+\infty} \exp \left(-i \alpha_{1}\left(x_{1}-t\right)\right) \cos \frac{\alpha_{1}}{M}\left(t^{2}-M^{2} r_{0}^{2}\right)^{1 / 2} d \alpha_{1}=\pi\left\{\delta\left[x_{1}-t+\frac{1}{M}\left(t^{2}-M^{2} r_{0}^{2}\right)^{1 / 2}\right]\right. \\
\left.+\delta\left[x_{1}-t-\frac{1}{M}\left(t^{2}-M^{2} r_{0}^{2}\right)^{1 / 2}\right]\right\}, \quad \delta(x-c)+\delta(x+c)=2 c \delta\left(x^{2}-c^{2}\right)
\end{gathered}
$$

obtained from [6] and [5]. We get

$$
\begin{aligned}
& \overline{\mathrm{f}}^{-1}\left(\frac{\exp \left(i \alpha_{1} t\right)}{|\boldsymbol{\alpha}|} \sin \frac{|\boldsymbol{\alpha}|}{M} t\right) \\
& =\frac{1}{4 \pi^{2}} \int^{+\infty} \exp \left(-j \alpha_{1}\left(x_{1}-t\right)\right) d \alpha_{1} \int_{0}^{\infty} \frac{J_{0}\left(\rho r_{0}\right) \rho \sin t M^{-1}\left(\rho^{2}+\alpha_{1}^{2}\right)^{1 / 2}}{\left(\rho^{2}+\alpha_{1}^{2}\right)^{1 / 2}} d \rho
\end{aligned}
$$




$$
\begin{aligned}
& =\frac{H\left(t-M r_{0}\right)}{2 \pi} \delta\left[\left(x_{1}-t\right)^{2}+r_{0}^{2}-\frac{t^{2}}{M^{2}}\right]=\frac{M H\left(t-M r_{0}\right)}{4 \pi t} \delta\left\{\left[\left(x_{1}-t\right)^{2}+r_{0}^{2}\right]^{1 / 2}-M^{-1} t\right\} \\
& =\frac{M}{4 \pi t} \delta\left\{M^{-1} t-\left[\left(x_{1}-t\right)^{2}+r_{0}^{2}\right]^{1 / 2}\right\} .
\end{aligned}
$$

Using the same change of variables we have

$$
\begin{aligned}
& \tilde{f}^{-1}\left(\frac{\exp \left(i \alpha_{1} t\right)}{|\alpha|^{2}}\right)=\frac{1}{4 \pi^{2}} \int_{-\infty}^{+\infty} \exp \left(i \alpha_{1}\left(t-x_{1}\right)\right) d \alpha_{1} \int_{0}^{\infty} \frac{J_{0}\left(\rho r_{0}\right) \rho d \rho}{\alpha_{1}^{2}+\rho^{2}} \\
& =\frac{1}{2 \pi^{2}} \int_{0}^{\infty} K_{0}\left(r_{0} \alpha_{1}\right) \cos \alpha_{1}\left(t-x_{1}\right) d \alpha_{1}=\frac{1}{4 \pi}\left[\left(t-x_{1}\right)^{2}+r_{0}^{2}\right]^{-1 / 2}, \\
& \tilde{f^{-1}}\left(\frac{\exp \left(i \alpha_{1} t\right)}{|\alpha|^{2}} \cos \frac{|\alpha|}{M} t\right)=\frac{1}{4 \pi^{2}} \int_{-\infty}^{+\infty} I \exp \left(i \alpha_{1}\left(t-x_{1}\right)\right) d \alpha_{1}
\end{aligned}
$$

where

$$
I(t)=\int_{0}^{\infty} \frac{J_{0}\left(\rho r_{0}\right)}{\rho^{2}+\alpha_{1}^{2}} \cos \frac{|\alpha|}{M} t d \rho
$$

Since the integral $I(t)$ is not given in tables, we shall calculate it here. Obviously we have

$$
\begin{gathered}
I(0)=K_{0}\left(\left|\alpha_{1}\right| r_{0}\right), \quad I^{\prime}(t)=-\frac{H\left(t-M r_{0}\right)}{\left(t^{2}-M^{2} r_{0}^{2}\right)^{1 / 2}} \cos \frac{\alpha_{1}}{M}\left(t^{2}-M^{2} r_{0}^{2}\right)^{1 / 2}, \\
I(t)=I(0)+\int_{0}^{t} I^{\prime}(\tau) d \tau=K_{0}\left(\left|\alpha_{1}\right| r_{0}\right)-H\left(t-M r_{0}\right) \int_{M r_{0}}^{t} \frac{\cos \alpha_{1} M^{-1}\left(\tau^{2}-M^{2} r_{0}^{2}\right)^{1 / 2}}{\left(\tau^{2}-M^{2} r_{0}^{2}\right)^{1 / 2}} d \tau,
\end{gathered}
$$

and consequently

$$
\begin{aligned}
\tilde{f}^{-1}\left(\frac{e^{i \alpha_{1} t}}{|\alpha|^{2}}\left(1-\cos \frac{|\alpha|}{M} t\right)\right)=\frac{H\left(t-M r_{0}\right)}{4 \pi} \int_{M r_{0}}^{t} \frac{d \tau}{\left(\tau^{2}-M^{2} r_{0}^{2}\right)^{1 / 2}} \\
\cdot\left\{\delta\left[x_{1}-t+\frac{1}{M}\left(\tau^{2}-M^{2} r_{0}^{2}\right)^{1 / 2}\right]+\delta\left[x_{1}-t-\frac{1}{M}\left(\tau^{2}-M^{2} r_{0}^{2}\right)^{1 / 2}\right]\right\} \\
=\frac{H\left(t-M_{0}\right)}{2 \pi M} \int_{M r_{0}}^{t} \delta\left[\left(x_{1}-t\right)^{2}+r_{0}^{2}-\frac{\tau^{2}}{M^{2}}\right] d \tau \\
=\frac{H\left(t-M r_{0}\right)}{4 \pi} \int_{M r_{0}}^{t} \frac{1}{\tau} \delta\left\{M^{-1} \tau-\left[\left(x_{1}-t\right)^{2}+r_{0}^{2}\right]^{1 / 2}\right\} d \tau .
\end{aligned}
$$

The final integral is non-vanishing only if $M\left[\left(x_{1}-t\right)^{2}+r_{0}^{2}\right]^{1 / 2}<t$, the inequality $r_{0}<\left[\left(x_{1}\right.\right.$ $\left.-r)^{2}+r_{0}^{2}\right]^{1 / 2}$ being always fulfilled. In these conditions, the factor $H\left(t-M r_{0}\right)$ becomes superfluous and consequently formula (A.3) is obtained.

\section{REFERENCES}

[1] L. Dragos, Fundamental solutions in fuid mechanics, ZA MP 1978

[2] L. Dragos, La matrice fondamentale pour les équations des fluides idéaux, J. Mécanique 1978

[3] L. Dragos, Solutions fondamentales dans la mécanique des fuides visqueux en mouvement uniforme, Rev. Roum. Math. Pures et Appl. 1979, No. 1.

[4] L. Dragos, Fundamental solutions in thermoelasticity, Acta Mechanica 1978

[5] I. Stakgold, Boundary value problems of mathematical physics, Vol. II, MacMillan, N.Y. 1968

[6] E. Erdelyí, (ed.), Tables of integral transforms, McGraw-Hill, 1954 